ISSN 1112-9867

http://www.jfas.info

\title{
CLASSIFICATION OF STUDENTS' IQ LEVEL USING EEG-BASED INTELLIGENCE CLASSIFIER MODEL
}

\author{
M. M. Anoor ${ }^{1}$, K. H. K. Anwar ${ }^{1}$, M. S. A. M. Ali ${ }^{2}$ and A. H. Jahidin ${ }^{1, *}$ \\ ${ }^{1}$ Centre for Foundation Studies in Science, University of Malaya, Kuala Lumpur, Malaysia \\ ${ }^{2}$ Faculty of Electrical Engineering, Universiti Teknologi MARA, Shah Alam, Selangor, \\ Malaysia
}

Published online: 17 October 2017

\begin{abstract}
Intelligence quotient (IQ) is used to measure the composite mental constituents of cognitive performance. Conventional methods for measuring IQ however are exposed to biasness issues. An alternative solution to classify IQ levels via electroencephalogram (EEG) based intelligence classifier model is proposed. 50 students from foundation program have participated in the pilot study. Resting EEG is recorded from the left prefrontal cortex. The signal is initially pre-processed for noise removal. Next, the extracted power ratio features are fed to the previously established classification model, enabling it to classify IQ level for each sample. Majority of the samples are classified as high IQ. Nevertheless, the pattern of theta, alpha and beta ratio have provided means of output validation via the Neural Efficiency Hypothesis of intelligence and alpha suppression theory.
\end{abstract}

Keywords: EEG; brainwave; IQ; power ratio; classifier.

Author Correspondence, e-mail: aisyah23@um.edu.my

doi: http://dx.doi.org/10.4314/jfas.v9i5s.48 


\section{INTRODUCTION}

Cognitive ability, often associated with intelligence is described as the capability of the brain to process information. It is the ability of individuals to comprehend complex ideas, participate in diverse forms of reasoning, efficiently adapt to the surroundings, learn from experience and solve problems through thinking [1]. Individual differences in intelligence are among the most vigorous observations in psychology and are stable in rank order throughout the development and lasts over long duration of time [2]. Numerous psychometric assessment methods have been developed to measure individual ability on different facets of intelligence. These include verbal reasoning, working memory, non-reasoning ability and mental rotation [3]. Thus far, intelligence quotient (IQ) has been acknowledged as the best predictor of the cognitive ability as it essentially summarizes all other constituent of mental constructs [4]. Apart from psychometric tests, a new method for assessing IQ via the electroencephalogram (EEG) has recently been proposed [5-7].

EEG is a non-invasive technique that records electrical signal from aggregated population of neurons in the brain. This is realized through bio-potential electrodes that captures the brainwaves from the scalp surface. The EEG is comprised of four major frequency bands.Delta wave ranges from $0.5 \mathrm{~Hz}$ to $4 \mathrm{~Hz}$, theta wave from $4 \mathrm{~Hz}$ to $8 \mathrm{~Hz}$, alpha wave from $8 \mathrm{~Hz}$ to $13 \mathrm{~Hz}$ and beta wave from $13 \mathrm{~Hz}$ to $30 \mathrm{~Hz}$. Each of these EEG bands holdexclusive information relating to specific brain processes [8]. Delta and theta waves are each commonly associated with deep and light sleep. Meanwhile, alpha waves are dominant when the brain is in tonic resting state. When the brain undergoes intense mental activity such as thinking, the synchronous activation of neuron population deteriorates and is replaced with the higher frequency beta waves [9]. The executive functions of the brain are performed by the prefrontal cortex. These include the ability to make rational decisions, plan and anticipate the future, shaping of motivational directed behavior and regulating emotions. The left side of prefrontal cortex is specialized for sequential and logic processes, while the right side is more involved in emotional abilities and social interactions [10].

The Neural Efficiency Hypothesis of intelligence has established that the brain of brighter individuals is used more efficiently in cognitively demanding tasks than those of the lessintelligent ones. The much localized brain activation during mental engagement is 
reflected in relatively lower glucose consumption in brighter individuals [3]. Their higher alpha power[11] indicates reduced cortical noise and higher level of perception and attentional state [12].Meanwhile, deviations from symmetrical hemispheric activities in less intelligent individuals also suggest a less efficient network configuration in the brain [13-14]. Similar observations have been recorded using various brain imaging modalities over an extensive range of experimental settings [3].

The conventional psychometric tests are exposed to several barriers in terms of language, misinterpretation of context and time constraints. These would indirectly affect the accuracy of assessed IQ [15]. To overcome such limitations, this paper proposes an implementation of an alternative method via brainwaves. An EEG-based IQ classifier modelwhich was developed earlier [16] will classify IQ levels of students from Centre for Foundation Studies in Science, University of Malaya. The study aims to examine the relationship between the classified IQ with theta, alpha and beta waves from the left prefrontal cortex.

\section{METHODOLOGY}

The framework of research methods involved in this study is shown in Fig. 1. The work comprise of sample selection and EEG acquisition, signal pre-processing and filtering, power ratio extraction and IQ level prediction using EEG-based intelligence classification model.

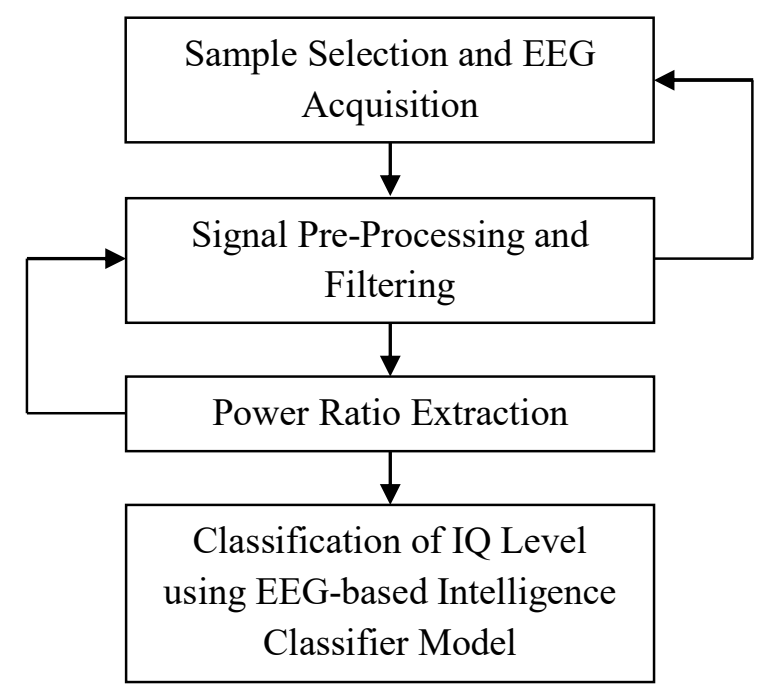

Fig.1. Flowchart of IQ level classification using the EEG-based intelligence classifier model 


\subsection{EEG Data Acquisition}

A total of 50 healthy students from Centre for Foundation Studies in Science, University of Malaya (mean age $/$ standard deviation $=18.1 / 0.24$, range $=18$ to 19 years) has volunteered in this pilot study. Samples comprised of 25 males and 25 females. All samples are informed on the experimental procedure and have given written consent prior to data collection.EEG is recorded from scalp location Fp1 (left prefrontal cortex) using Neurosky Mobile Wave headset. As shown in Fig. 2, the reference is attached to the left earlobe. The positioning conforms to the International 10-20 System for electrode placements. The sampling frequency of the device is $512 \mathrm{~Hz}$.

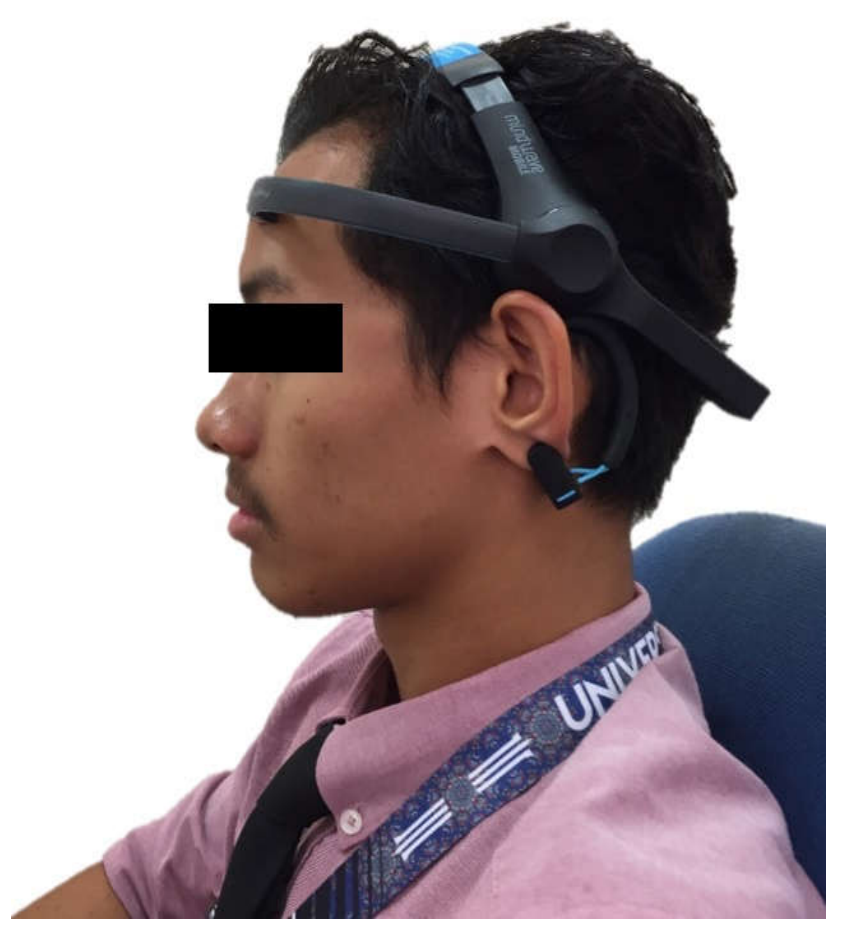

Fig.2.Placement of Neurosky Mobile Wave headset

The protocol requires the subjects to relax in seated position with both eyes closed. Recording is performed in a quiet room to avoid disturbance from external noises. Each session lasts for approximately 3 minutes. Both the real-time attention and meditation readings are monitored throughout the recording session.

\subsection{Signal Pre-Processing, Filtering and Feature Extraction}

The raw signal is initially pre-processed using MATLAB 2015a. EEG epochs exceeding \pm 100 $\mu \mathrm{V}$ are regarded as electrooculogram noises and thus, automatically rejected [17]. Further analysis, the signal is limited to duration of 2 minutes 30 seconds [18-19]. The pre-processed 
signal is then segregated into theta, alpha and beta waves viaequiripple band-pass filters. Power spectral density (PSD) for each frequency bandis then acquired via Welch's method. Subsequently, energy spectral density (ESD) is computed as energy distribution under the PSD curve [20]. The information between the EEG bands are then normalized using power ratio relationships expressed by Equation (1)-(3).

$$
\begin{aligned}
& \text { Theta Ratio }=\frac{\theta}{\theta+\alpha} \\
& \text { Alpha Ratio }=\frac{\alpha}{\alpha+\beta} \\
& \text { Beta Ratio }=\frac{\beta}{\alpha+\beta}
\end{aligned}
$$

\subsection{EEG-Based Intelligence Classification Model}

Artificial neural network has been implemented in earlier studies to establish the EEG-based intelligence classifier model. It was developed using power ratio features based on 120 samples [14]. In the present study, the obtained power ratio features for each of the samples are fed to the network input; enabling the model to classify IQ levels as either low, medium or high. Table 1 shows the IQ levels, its range of output and the corresponding IQ indexes.

Table 1. IQ levels, range of output and IQ indexes

\begin{tabular}{ccc}
\hline IQ Level & Range of Output & IQ Index \\
\hline Low & $0.5<$ IQ Index $<1.5$ & 1 \\
Medium & $1.5<$ IQ Index $<2.5$ & 2 \\
High & $2.5<$ IQ Index $<3.5$ & 3 \\
\hline
\end{tabular}

\section{RESULTS AND DISCUSSION}

\subsection{Classification of IQ Levels Using EEG-Based Intelligence Classifier Model}

Generally, the model has successfully classified IQ levels for all 50 samples. None has been categorized as unclassified. 47 samples are classified as high IQ, followed by 3 samples in the medium IQ group. None has been classified as low IQ. The mean of IQ index and standard deviation for 50 samples is 2.93 and 0.29 respectively. Distribution of samples based on the classified IQ and gender is shown in Fig. 3. 
30

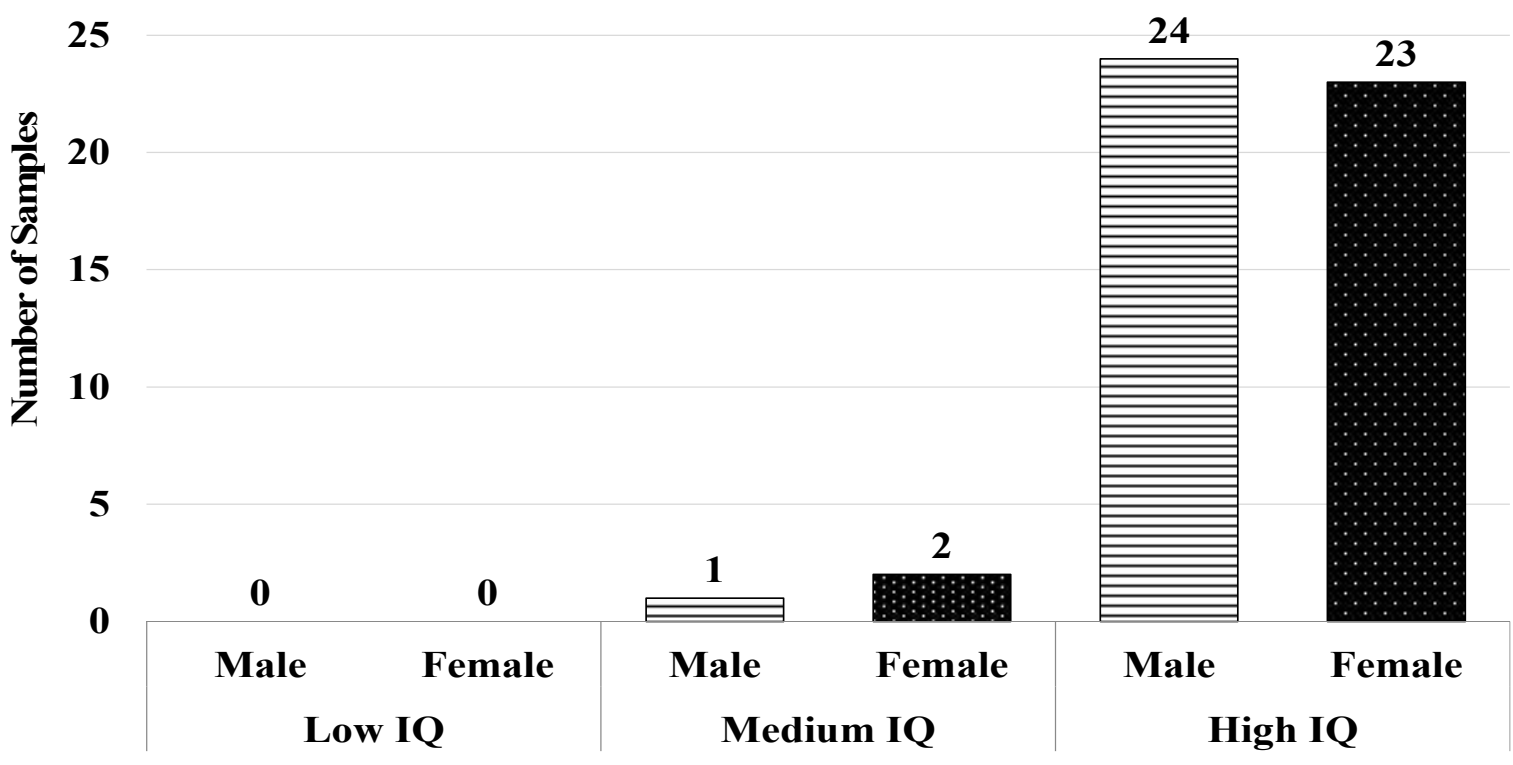

Fig.3.Distribution of samples based on the classified IQ levels and gender

The skewed distribution towards high IQ level is to be expected as majority of students that are selected into the Centre for Foundation Studies in Science are high achievers of the Malaysian Education Certificate. Hence, the pattern is in agreement with their excellent qualifications that are required for enrolling into the foundation program.

\subsection{Correlation of Mean Power Ratio Pattern with IQ Levels}

None of the samples have been classified as low IQ. Therefore, the ensuing discussion only focuses on medium and high IQ groups. The pattern of mean theta, alpha and beta ratio according to gender groups is shown in Fig. 4. Overall, both genders have demonstrated similar pattern variation between the two IQ levels. The mean alpha ratio for high IQ group is comparatively higher than the medium IQ group. Findings are in agreement with the Neural Efficiency Hypothesis which states that cortical activation is less intense in brighter individuals. Their relatively lower beta ratio also reflects the reduced cortical noise which resulted in increased level of perception and attentional state. The pattern of theta ratio further explains the reciprocal relationship between both theta and alpha bands. In alpha suppression theory, synchronization of theta waves results in the desynchronization of alpha waves [12]. These are reflected in higher theta ratio for medium IQ group. 


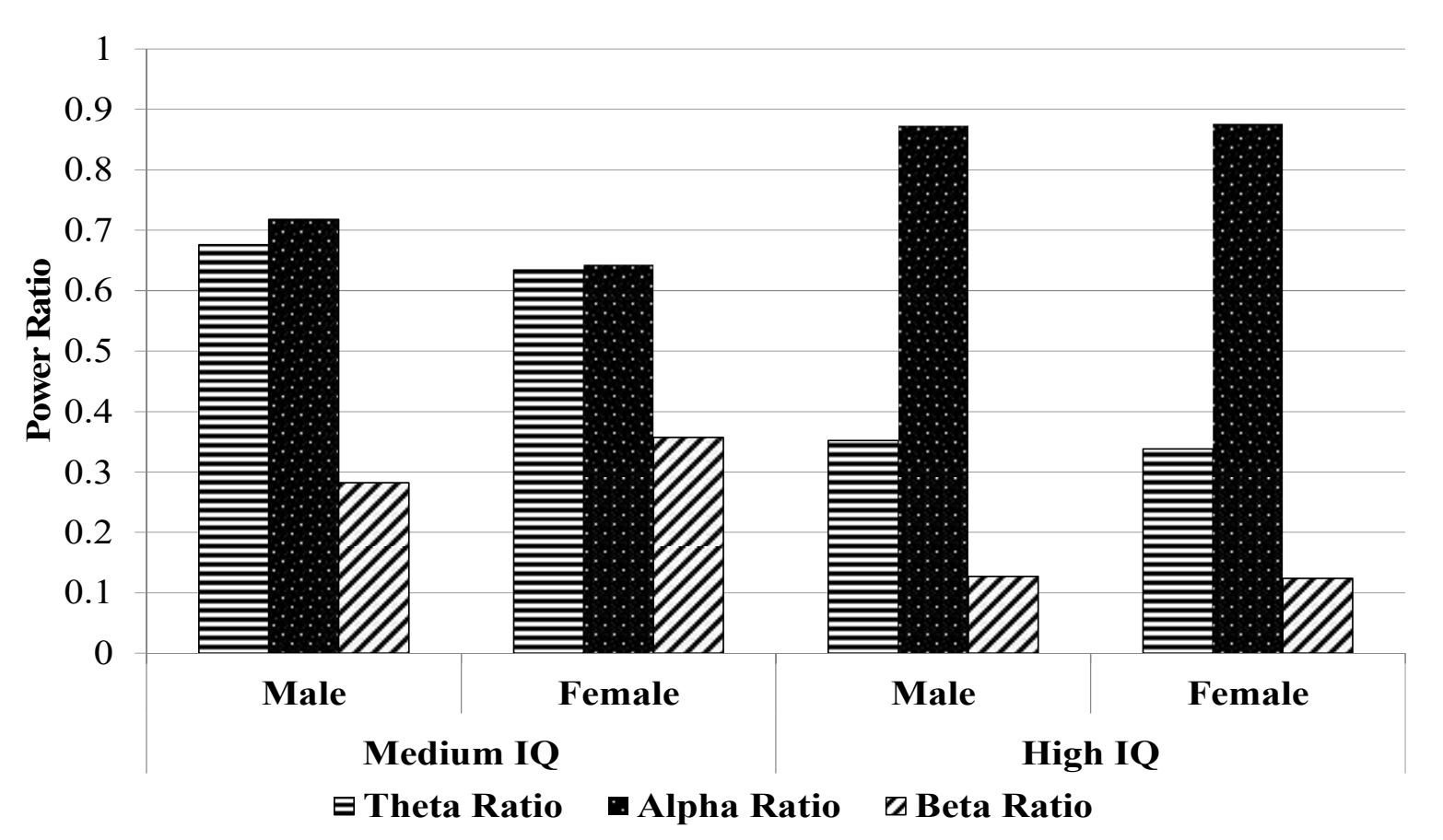

Fig.4.Comparison of mean power ratio features between medium and high IQ

Table 2 summarizes the mean power ratio values and pattern variations for both medium and high IQ groups.

Table 2.Mean power ratio and pattern variation for medium and high IQ groups

\begin{tabular}{ccccc}
\hline Gender & Power Ratio & \multicolumn{2}{c}{ IQ Level } & Trend from \\
& & Medium & High & Medium to High IQ \\
\hline \multirow{2}{*}{ Male } & Theta & 0.68 & 0.35 & Decreasing \\
& Alpha & 0.72 & 0.87 & Increasing \\
& Beta & 0.28 & 0.13 & Decreasing \\
\multirow{2}{*}{ Female } & Theta & 0.63 & 0.34 & Decreasing \\
& Alpha & 0.64 & 0.88 & Increasing \\
& Beta & 0.36 & 0.12 & Decreasing
\end{tabular}

Findings revealed that individuals with high IQ level exhibit relatively higher alpha, but lower beta and theta ratio. Meanwhile, those with medium IQ level will demonstrate inversed power ratio pattern with lower alpha but higher beta and theta ratio. Table 3 further shows the percentage of difference between alpha and beta ratio, as well as alpha and theta ratio. These are significant as it reflect the varying levels of alpha dominance for different IQ levels. 
Table 3.Percentage of difference between alpha and beta ratio, as well as alpha and theta ratio for each IQ group

\begin{tabular}{ccccc}
\hline \multirow{2}{*}{ Gender } & \multicolumn{2}{c}{ Alpha-Beta } & \multicolumn{2}{c}{ Alpha-Theta } \\
\cline { 2 - 5 } & Medium IQ & High IQ & Medium IQ & High IQ \\
\hline Male & $61.10 \%$ & $85.06 \%$ & $5.56 \%$ & $59.77 \%$ \\
\hline Female & $43.75 \%$ & $86.36 \%$ & $1.56 \%$ & $61.36 \%$ \\
\hline
\end{tabular}

The percentage of difference is greater for the high IQ group. This indicates a higher level of alpha dominance over beta and theta ratio features. For medium IQ group however, the percentage difference deteriorates. These are valid as cortical noise is expected to be higher and the reduced alpha power enables synchronization of lower frequency brainwaves, which results in higher theta ratio for medium IQ group.

\section{CONCLUSION}

The study has successfully implemented the EEG-based [21] intelligence classifier model to classify IQ levels of students from the Centre for Foundation Studies in Science, University of Malaya. Majority of the samples have been classified into the high IQ group. These are expected as the students are high achievers of the Malaysian Education Certificate. Excellent academic qualification is a prerequisite for them to enroll into the foundation program. Meanwhile, findings on theta, alpha and beta ratio patterns are in agreement with the Neural Efficiency Hypothesis of intelligence which outlines brighter individuals as being able to use the brain in relatively more efficient manner. Results for high IQ group also suggest greater alpha dominance compared to the medium IQ due to the lower cortical noise and reduced alpha suppression ability. Conclusively, the EEG-based [22] intelligence classifier model has been successfully implemented to classify IQ levels and findings are validated by established theories of human intelligence.

\section{ACKNOWLEDGEMENTS}

This research is supported by the Centre for Foundation Studies in Science, University of Malaya (BK035-2016) and Institute of Research Management and Innovation, Universiti Teknologi MARA, Malaysia (600-IRMI/MyRA 5/3/LESTARI (015/2017)). 


\section{REFERENCES}

[1] Gray J R, Thompson P M., Neurobiology of intelligence: Science and ethics. Nature Reviews Neuroscience, 2004, 5(6):471-482

[2] Lubinski D. Introduction to the special section on cognitive abilities: 100 years after Spearman's (1904)"'General intelligence,' objectively determined and measured". Journal of Personality and Social Psychology, 2004, 86(1):96-111

[3] Neubauer A C, Fink A. Intelligence and neural efficiency. Neuroscience and Biobehavioral Reviews, 2009, 33(7):1004-1023

[4] Lee T W, Wu Y T, Yu Y W Y, Wu H C, Chen T J. A smarter brain is associated with stronger neural interaction in healthy young females: A resting EEG coherence study. Intelligence, 2012, 40(1):38-48

[5] Jahidin A H, Taib M N, Md Tahir N, Megat A M S A, Lias S, Fuad N, Omar W R W. Brainwave sub-band power ratio characteristics in intelligence assessment.In IEEE Control and System Graduate Research Colloquium, 2012, pp. 318-321

[6] Jahidin A H, Taib M N, Megat A M S A, Md Tahir N, Lias S, Haron M H, Isa R M, Omar W R W, Fuad N. Evaluation of brainwave sub-band spectral centroid in human intelligence.In IEEE 9th International Colloquium on Signal Processing and its Applications, 2013, pp. 295-298

[7] Jahidin A H, Taib M N, Md Tahir N, Megat A M S A. IQ classification via brainwave features: Review on artificial intelligence techniques. International Journal of Electrical and Computer Engineering, 2015, 5(1):84-91

[8] Mathewson K J, Jetha M K, Drmic I E, Bryson S E, Goldberg J O, Schmidt L A. Regional EEG alpha power, coherence, and behavioral symptomatology in autism spectrum disorder. Clinical Neurophysiology, 2012, 123(9):1798-1809

[9] Webster J. G. Medical instrumentation: Application and design. New Jersey: Wiley, 2009

[10] van der Knaap L J, van der Ham I J M. How does the corpus callosum mediate interhemispheric transfer? A review. Behavioural Brain Research, 2011, 223(1):211-221

[11] Doppelmayr M, Klimesch W, Stadler W, Pöllhuber D, Heine C. EEG alpha power and intelligence. Intelligence, 2002, 30(3):289-302

[12] Foxe J J, Snyder A C. The role of alpha-band brain oscillations as a sensory suppression 
mechanism during selective attention. Frontiers in Psychology, 2011, 2:1-13

[13] Jahidin A H, Taib M N, Md Tahir N, Megat A M S A, Lias S. Asymmetry pattern of resting EEG for different IQ levels.In 9th International Conference on Cognitive Science, 2013, pp. 246-251

[14] Jahidin A H, Megat A M S A, Taib M N, Md T N, Mohd Y A I. Cross-relational study between intelligence and brain asymmetry abilities using EEG-based IQ classification model. Journal of Theoretical and Applied Information Technology, 2015, 81(1):43-50

[15] Colom R, Karama S, Jung R E, Haier R J. Human intelligence and brain networks. Dialogues in Clinical Neuroscience, 2010, 12(4):489-501

[16] Jahidin A H, Megat A M S A, Taib M N, Md T N, Yassin I M, Lias S. Classification of intelligence quotient via brainwave sub-band power ratio features and artificial neural network. Computer Methods and Programs in Biomedicine, 2014, 114(1):50-59

[17] Schlögl A, Keinrath C, Zimmermann D, Scherer R, Leeb R, Pfurtscheller G. A fully automated correction method of EOG artifacts in EEG recordings. Clinical Neurophysiology, 2007, 118(1):98-104

[18] Megat A M S A, Taib M N, Md T N, Jahidin A H, Yassin I M. EEG sub-band spectral centroid frequencies extraction based on Hamming and equiripple filters: A comparative study.In IEEE 10th International Colloquium on Signal Processing and its Applications, 2014, pp. 199-203

[19]Megat A M S A, Jahidin A H, Taib M N, Md T N. EEG sub-band spectral centroid frequency and amplitude ratio features: A comparative study in learning style classification. Jurnal Teknologi, 2016, 78(2):15-23

[20] Jahidin A H, Taib M N, Md T N, Megat A M S A, Yassin I M, Lias S, Isa R M, Omar W R W, Fuad N. Classification of intelligence quotient using EEG sub-band power ratio and ANN during mental task.In IEEE Conference on Systems, Process and Control, 2013, pp. 204-208

[21] Zaman F H, Shukur N A, Hamzah N, Zaini N M, Rizman Z I. Performance of modified power spectral density features in EEG signal classification. Journal of Fundamental and Applied Sciences, 2017, 9(3S):830-843

[22] Ismail W W, Hanif M, Mohamed S B, Hamzah N, Rizman Z I. Human emotion detection 
via brain waves study by using electroencephalogram (EEG). International Journal on Advanced Science, Engineering and Information Technology, 2016, 6(6):1005-1011

\section{How to cite this article:}

Anoor M M, Khairul AnwarK H, Megat Ali M S A and Jahidin A H. Classification of students' IQ level using EEG-based intelligence classifier model. J. Fundam. Appl. Sci., 2017, 9(5S), 684-694. 